\title{
PERIODIC ORBITS IN PLANAR SYSTEMS MODELLING NEURAL ACTIVITY
}

\author{
BY \\ ROBERT E. KOOIJ (Department of Applied Mathematical Analysis, Delft University of \\ Technology, Information Technology and Systems, Mekelweg 4, 2628 CD Delft, The Netherlands) \\ AND \\ FOTIOS GIANNAKOPOULOS (GMD-German Research Center for Information Technology, \\ Schloss Birlinghoven, 53754 Sankt Augustin, Germany)
}

\begin{abstract}
In this paper we will prove certain properties of a planar dynamical system modelling the neural activity of a network consisting of two neurons. At first we show that for a certain region in parameter space (such that there exist three equilibria) the dynamical system has no periodic orbits. To this end we need a new criterion for the nonexistence of limit cycles in a system of Liénard type (Lemma 3.1). Next we derive conditions under which our model system has exactly one periodic orbit, which will be a stable limit cycle. Finally, we cover a part of the parameter space where we can prove that the dynamical system has three equilibria such that around two of the equilibria at most one limit cycle can exist.
\end{abstract}

1. Introduction. In this work we study the number of periodic orbits of a mathematical model for neural activity of a small network consisting of two nerve cells; see Fig. 1.1. The model takes the following important neurophysiological properties into account (for details see [14]).

Spatial and temporal integration. A nerve cell collects incoming signals from other cells on its dendrites which sum up both temporally and spatially to the total potential at the cell body.

Nonlinear signal generation. The neuron will generate an output signal, which is a (nonlinear) increasing function of the total potential.

Excitation and inhibition. Neurons can be classified as excitatory and inhibitory neurons. Excitatory neurons cause a positive electrical membrane potential difference at the next neuron, whereas inhibitory ones cause a negative potential difference.

The network considered consists of an excitatory and an inhibitory neuron; see Fig.

Received August 7, 1997.

2000 Mathematics Subject Classification. Primary 34Cxx, 37-XX, 92Cxx.

The current address of the first author is KPN Research, 2260 AK Leidschendam, The Netherlands.

E-mail address: r.e.kooij@research.kpn.com

E-mail address: giannakopoulos@gmd.de 


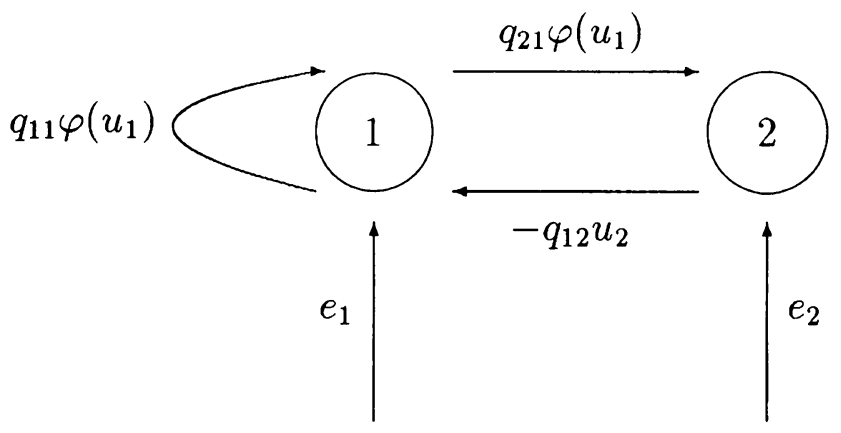

FIG. 1.1. Connection scheme of the neural network modelled by (1.1)

1.1. The mathematical model describing the dynamics of the network is given by (for details see [6]):

$$
\begin{aligned}
& \frac{d u_{1}}{d t}=-u_{1}+q_{11} \varphi\left(u_{1}\right)-q_{12} u_{2}+e_{1}, \\
& \frac{d u_{2}}{d t}=-u_{2}+q_{21} \varphi\left(u_{1}\right)+e_{2} .
\end{aligned}
$$

The functions and parameters occurring in system (1.1) have the following neurophysiological meaning.

- $u_{1}, u_{2}: \mathbb{R} \rightarrow \mathbb{R}$ denote the total potential of the excitatory and inhibitory neuron, respectively.

- $q_{i k}$ is a positive constant that represents the strength of the connection line from the $k$-th neuron to the $i$-th neuron (see Fig. 1.1). In this work we assume $q_{22}=0$, i.e., there is no self-inhibition.

- $\varphi: \mathbb{R} \rightarrow \mathbb{R}^{+}$is the transfer function that describes the activity generation of the 1-th (excitatory) neuron as a function of its total potential $u_{1}(t)$. In this work we choose (see Fig. 1.2):

$$
\varphi\left(u_{1}\right)=\frac{1}{1+\exp \left(-4 u_{1}\right)} .
$$

For the sake of simplicity the transfer function corresponding to the inhibitory neuron is assumed to be the identity.

- $e_{1}, e_{2}$ are external stimuli acting on the 1-th (excitatory) and 2-th (inhibitory) neuron, respectively.

The system (1.1) is a special case of an additive neural network of the form

$$
\begin{aligned}
& \frac{d u_{1}}{d t}=-u_{1}+q_{11} \varphi_{1}\left(u_{1}\right)-q_{12} \varphi_{2}\left(u_{2}\right)+e_{1}, \\
& \frac{d u_{2}}{d t}=-u_{2}+q_{21} \varphi_{1}\left(u_{1}\right)-q_{22} \varphi_{2}\left(u_{2}\right)+e_{2} ;
\end{aligned}
$$

see Cowan and Ermentrout [3], with $\varphi_{1}=\varphi, \varphi_{2}=\operatorname{id} \mathbb{R}_{\mathbb{R}}$, and $q_{22}=0$. If $q_{11} q_{22}-q_{12} q_{21} \neq 0$, by setting

$$
u_{i}=q_{i 1} v_{1}-q_{i 2} v_{2}+e_{i}, \quad i=1,2,
$$




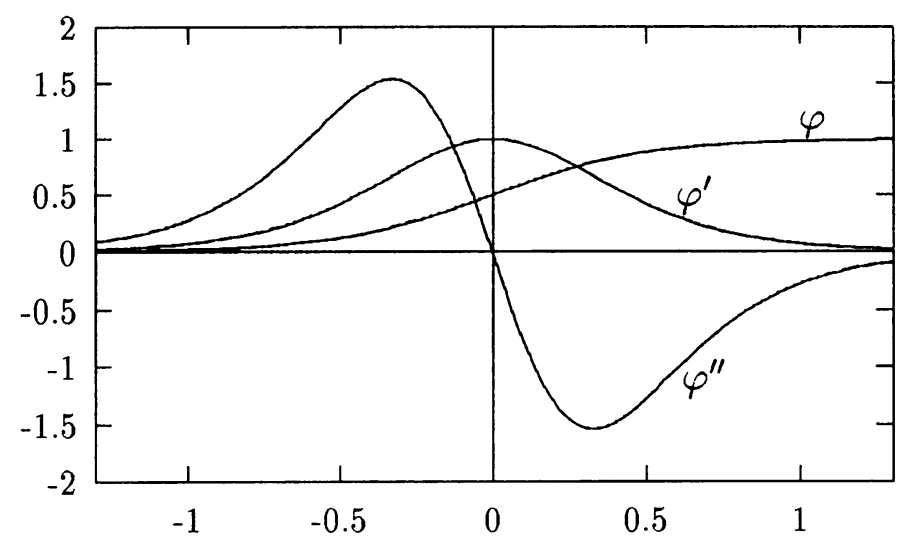

FIG. 1.2. The function $\varphi(u)=\frac{1}{1+\exp (-4 u)}$ and its derivatives

the system (1.3) becomes

$$
\begin{aligned}
& \frac{d v_{1}}{d t}=-v_{1}+\varphi_{1}\left(q_{11} v_{1}-q_{12} v_{2}+e_{1}\right) \\
& \frac{d v_{2}}{d t}=-v_{2}+\varphi_{2}\left(q_{21} v_{1}-q_{22} v_{2}+e_{2}\right)
\end{aligned}
$$

which is a special case of the following system introduced by Wilson and Cowan (see $[17])$ :

$$
\begin{aligned}
& \frac{d v_{1}}{d t}=-v_{1}+\left(1-r_{1} v_{1}\right) \varphi_{1}\left(q_{11} v_{1}-q_{12} v_{2}+e_{1}\right), \\
& \frac{d v_{2}}{d t}=-v_{2}+\left(1-r_{2} v_{2}\right) \varphi_{2}\left(q_{21} v_{1}-q_{22} v_{2}+e_{2}\right),
\end{aligned}
$$

with $r_{1}=r_{2}=0$.

Neural networks of the Wilson-Cowan type (and thus additive networks, too) possess properties, which play an important role in the information processing in the brain. Two of these properties are the ability to carry out sustained oscillations and multistability (see [17], [3]). Neural circuits consisting of excitatory and inhibitory neurons arise in several regions of the brain, for instance, in the thalamus (see [16, p. 550]). They provide a possible mechanism of generation of rhythmic activity in the brain which has been observed in experiments (see [14], [16]). On the other hand, multistable neural networks can be viewed as models for associative memory (see [10], [9]). Attractors (for example, stable equilibria or limit cycles) can be interpreted as stored memories. In this context, the number of attractors characterizes the quality of the model. But not only the number of stable equilibria and periodic solutions is important. The number of unstable periodic solutions can also be of particular importance. For instance, unstable periodic orbits in planar dynamical systems define the boundaries of the attraction domains of attractors (see Fig. 1.6).

The first results on the existence of limit cycles and multiple stable equilibria for the system (1.6) have been proved by Wilson and Cowan [17]; see also [3] for a similar analysis of the system (1.3). In [2], using computer techniques, a bifurcation analysis of 


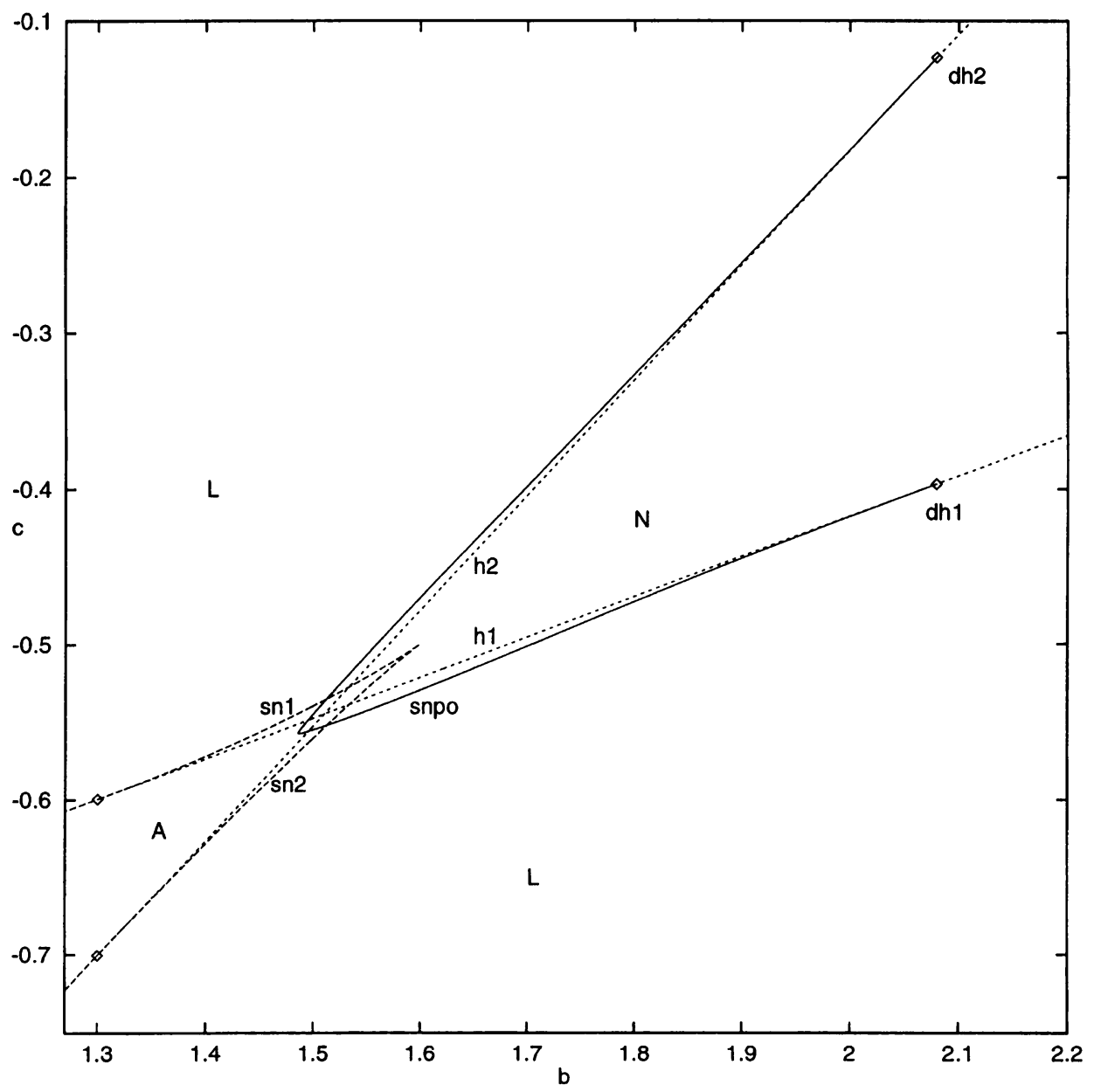

FIG. 1.3. The curves of saddle-node bifurcations $s n_{1}, s n_{2}$, Hopf bifurcations $h_{1}, h_{2}$ and saddle-node bifurcations of periodic orbits snpo. $d h_{1}, d h_{2}$ are Hopf bifurcation points of codimension $>1$. Taken (modified) from [6]. Regard $b=q_{12} q_{12}$ and $c=e_{1}-q_{12} e_{2}$.

the system (1.6) is provided with $e_{1}$ and $q_{21}$ as bifurcation parameters. In [9] bifurcation properties of the system (1.6) with $\varphi_{1}=\varphi_{2}$ are analyzed with $e_{1}$ and $e_{2}$ as bifurcation parameters.

In [6], the bifurcation properties of the system (1.1) for fixed $q_{11}$ are studied, when the negative feedback $b:=q_{12} q_{12}$ and total input $c:=e_{1}-q_{12} e_{2}$ acting on the excitatory neuron are varied. Using both local mathematical analysis and numerical techniques, the complete bifurcation diagram in the $(b, c)$-plane of the system (1.1) with fixed special value for the parameter $q_{11}$ is provided; see Figures 1.3, 1.4, 1.5, and 1.6.

The bifurcation diagram presented in [6] (see also Figures 1.3-1.6) is consistent with bifurcation theory (see [4]), but a rigorous verification of its completeness and correctness is a difficult mathematical problem. For instance, the co-existence of two large periodic orbits (i.e., periodic orbits surrounding three equilibria) and a small periodic orbit (i.e., 


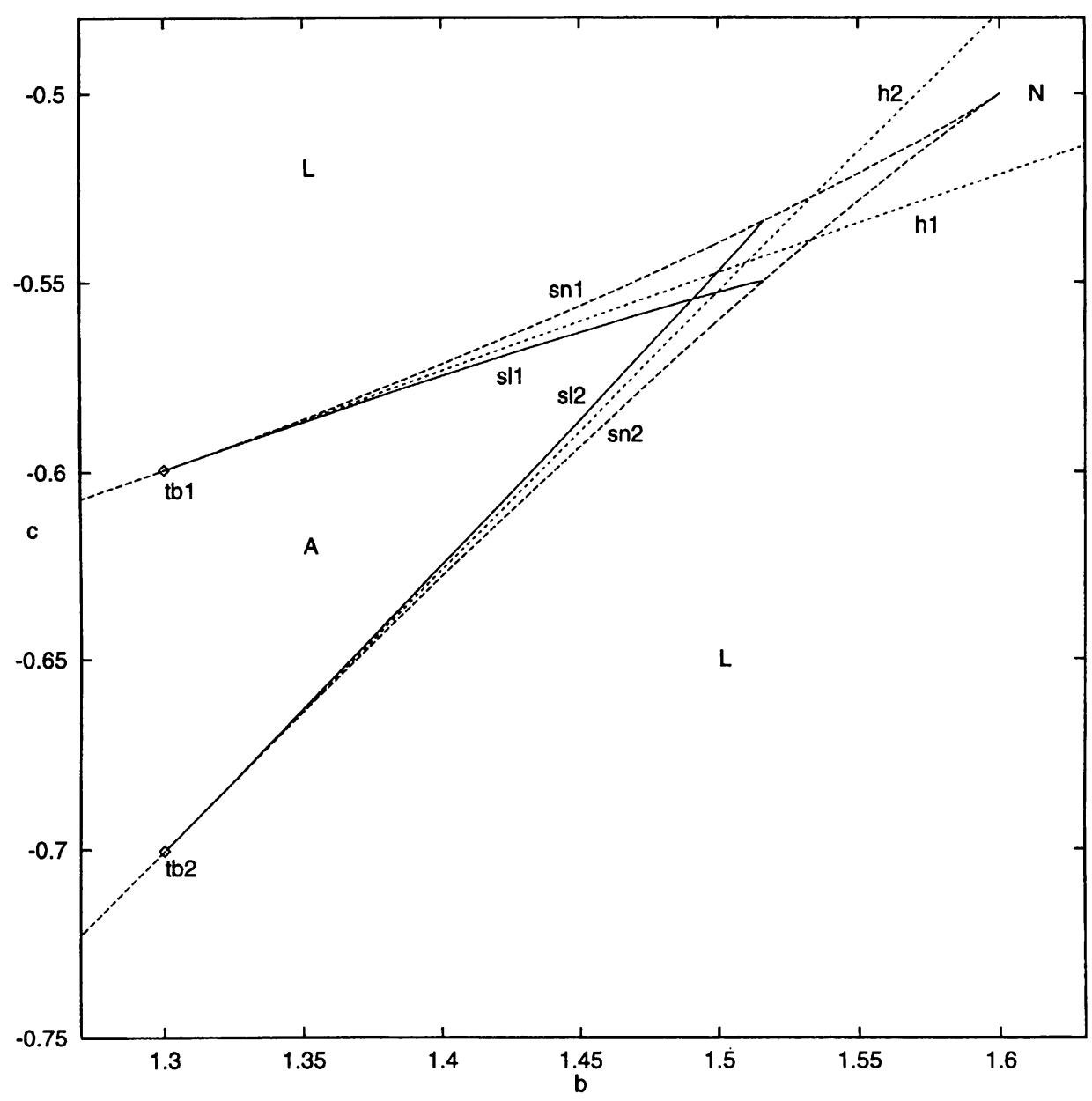

FIG. 1.4. The curves of saddle-node bifurcations $s n_{1}, s n_{2}$, Hopf bifurcations $h_{1}, h_{2}$ and the curves of saddle loops $s l_{1}, s l_{2}, t b_{1}, t b_{2}$ are Takens-Bogdanov bifurcation points. Taken (modified) from [6]. Regard $b=q_{12} q_{12}$ and $c=e_{1}-q_{12} e_{2}$.

a periodic orbit surrounding one equilibrium), and the co-existence of one large periodic orbit and two small periodic orbits of the system (1.1) with parameters $\left(q_{12} q_{12}, e_{1}-\right.$ $\left.q_{12} e_{2}\right) \in C$ and $\left(q_{12} q_{12}, e_{1}-q_{12} e_{2}\right) \in H$ (see Figures 1.5 and 1.6), respectively, is based upon numerical calculations. Rigorous proofs of the occurrence of such phenomena are still missing.

The objective of this work is to prove the correctness of the bifurcation diagram for some specific cases.

In this work we prove the following results:

- nonexistence of periodic orbits of system (1.1) for parameters $\left(q_{12} q_{12}, e_{1}-q_{12} e_{2}\right)$ in a large subset of $A$ (see Figures 1.5 and 1.6 (Theorem 4.1));

- uniqueness of periodic orbits for parameters $\left(q_{12} q_{12}, e_{1}-q_{12} e_{2}\right) \in N$ in the "symmetrical" case (see Figures 1.5 and 1.6 (Theorem 4.2)); 


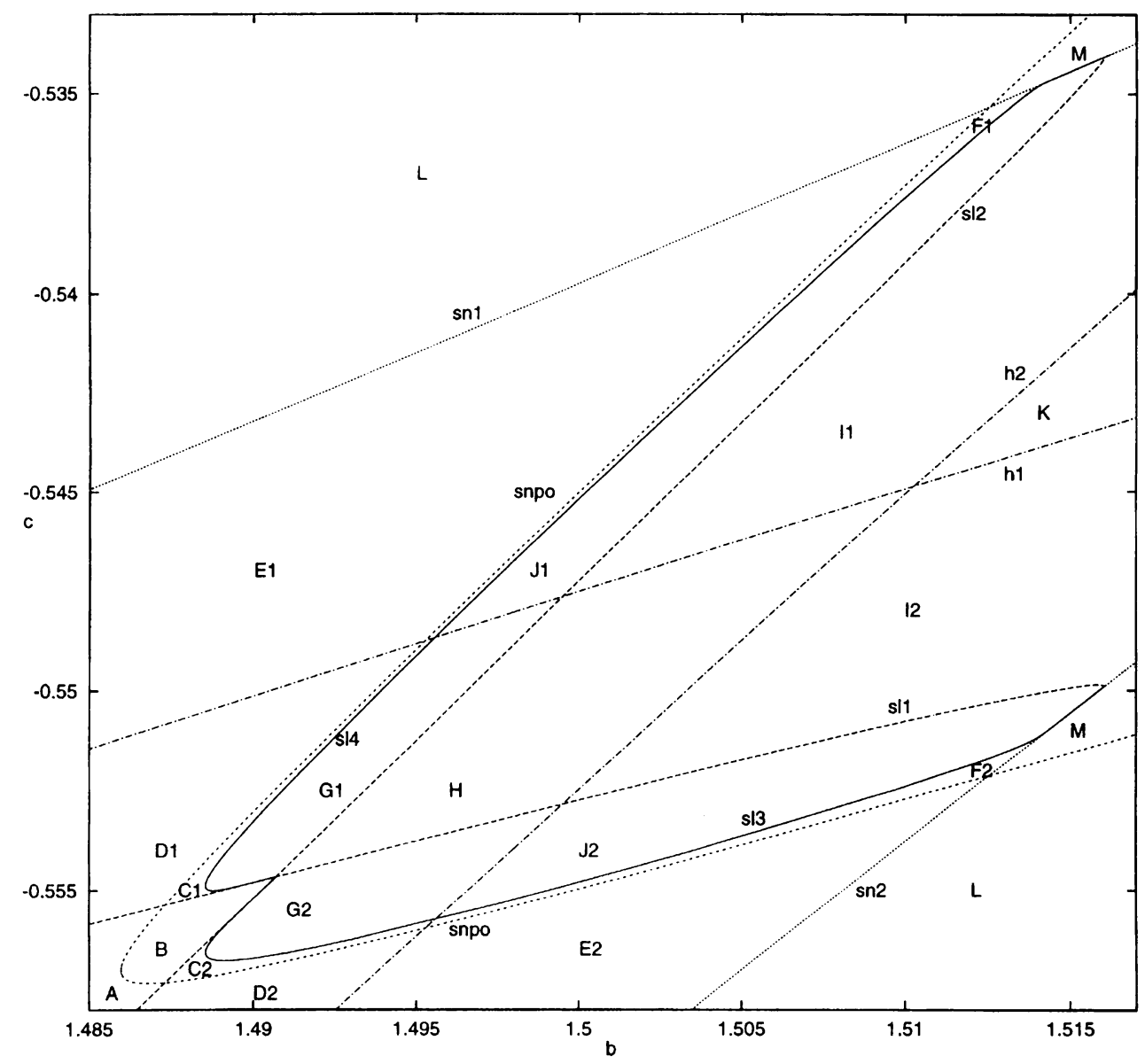

FIG. 1.5. A section of the bifurcation diagram of (1.1) (and thus (1.8)) consisting of the curves of Hopf bifurcations $h_{1}, h_{2}$, saddlenode bifurcations $s n_{1}, s n_{2}$, saddle-nodes of periodic orbits snpo and saddle-loops $s l_{1}, s l_{2}, s l_{3}, s l_{4}$. Taken (modified) from [6]. Regard $b=$ $q_{12} q_{12}$ and $c=e_{1}-q_{12} e_{2}$.

- uniqueness and nonexistence of two small periodic orbits for parameters $\left(q_{12} q_{12}\right.$, $\left.e_{1}-q_{12} e_{2}\right) \in H$ and $\left(q_{12} q_{12}, e_{1}-q_{12} e_{2}\right) \in K$ (see Figures 1.5 and 1.6, respectively, in the "symmetrical" case (Theorem 4.3)).

By the symmetrical case we mean parameters in the $(b, c)$-plane (regard $b=q_{12} q_{12}$ and $\left.c=e_{1}-q_{12} e_{2}\right)$ that are lying on the straight line given by the intersection point of the curves of the Hopf bifurcations $h_{1}, h_{2}$ and the intersection point of the curves of the saddle-node bifurcations $s n_{1}, s n_{2}$; see Fig. 1.3.

It can be shown that for the symmetrical case the solutions of system (1.1) are symmetric with respect to the equilibrium $\left(0, \frac{q_{21}}{2}+e_{2}\right)$. This means that if $(u(t), v(t))$ is a solution of system (1.1), then so is $\left(-u(t), q_{21}+2 e_{2}-v(t)\right)$.

Before we start with the analysis of the system (1.1), let us try to reduce the number of the parameters by using the following affine transformation. 

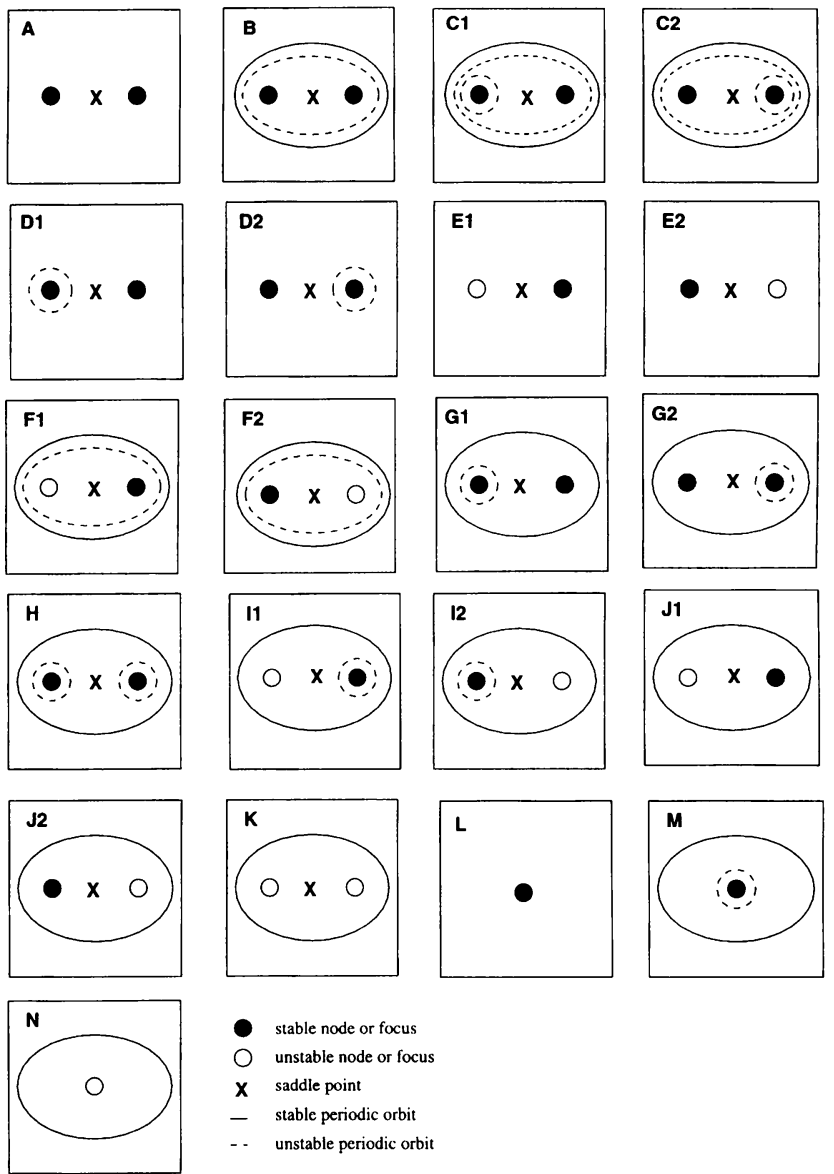

$$
\begin{array}{ll}
\text { - } & \text { stable node or focus } \\
\mathbf{X} & \text { unstable node or focus } \\
\text { - } & \text { saddle point } \\
\text { - - } & \text { stable periodic orbit } \\
& \text { unstable periodic orbit }
\end{array}
$$

FIG. 1.6. Simplified phase portraits corresponding to the regions of the diagrams in Figures 1.3 and 1.5. Taken (modified) from [6].

Setting

$$
u:=u_{1} \quad \text { and } \quad v:=\frac{1}{q_{21}}\left(u_{2}-e_{2}\right),
$$

the system (1.1) becomes

$$
\begin{aligned}
& \frac{d u}{d t}=-u+a \varphi(u)-b v+c \\
& \frac{d v}{d t}=-v+\varphi(u)
\end{aligned}
$$

where

$$
a:=q_{11}, \quad b:=q_{12} q_{21}, \quad c:=e_{1}-q_{12} e_{2} .
$$

Notice that the new system (1.8) is equivalent to the original system (1.1). The advantage of the new system (1.8) is that it possesses only three parameters. The physical meaning of the new parameters $a, b$ and $c$ is clear (see Fig. 1.1): $a$ describes the positive feedback, 
$b$ describes the negative feedback of the system, and $c$ describes the total external input acting on the 1-th neuron.

2. Preliminaries. In order to be able to state our results, we need some basic properties of the system (1.8). We start with the existence of equilibria.

It is easily verified that $(u, v) \in \mathbb{R}^{2}$ is an equilibrium of (1.8) if and only if

$$
\begin{aligned}
& c=g_{0}(u):=u-(a-b) \varphi(u), \\
& v=\varphi(u) .
\end{aligned}
$$

LEMMA 2.1 (Existence of equilibria).

(1) If $1 \geq a-b$, then there is a unique equilibrium of (1.8) for each $c \in \mathbb{R}$.

(2) If $1<a-b$, then there are $c_{1}, c_{2} \in \mathbb{R}$ with $c_{2}<c_{1}$ such that

(a) for $c \in\left(-\infty, c_{2}\right) \cup\left(c_{1},+\infty\right)$ there exists exactly one equilibrium,

(b) for $c \in\left\{c_{1}, c_{2}\right\}$ there exist exactly two equilibria,

(c) for $c \in\left(c_{2}, c_{1}\right)$ there exist exactly three equilibria.

Proof. Since

$$
\lim _{u \rightarrow \pm \infty} g_{0}(u)= \pm \infty
$$

system (1.8) has at least one equilibrium. For $1 \geq a-b$, the function $g_{0}$ is strictly increasing. This yields the uniqueness of the equilibrium. If $1<a-b$, then there are exactly two solutions $u_{01}, u_{02}$, with $u_{01}<0<u_{02}=-u_{01}$, of

$$
g_{0}^{\prime}(u)=1-(a-b) \varphi^{\prime}(u)=0
$$

such that $g_{0}$ is strictly increasing on $\left(-\infty, u_{01}\right]$ and $\left[u_{02},+\infty\right)$, and strictly decreasing on $\left[u_{01}, u_{02}\right]$; see Figures 2.1 and 2.2 .

With

$$
c_{i}:=g_{0}\left(u_{0 i}\right)=u_{0 i}-(a-b) \varphi\left(u_{0 i}\right), \quad i=1,2,
$$

the lemma is proved.

REMARK 2.1. For $1<a-b$, the two solutions $u_{01}, u_{02}$ of Eq. (2.3) are continuous functions of $b<a-1$. This implies that $c_{1}, c_{2}$ in Lemma 2.1 are also continuous functions

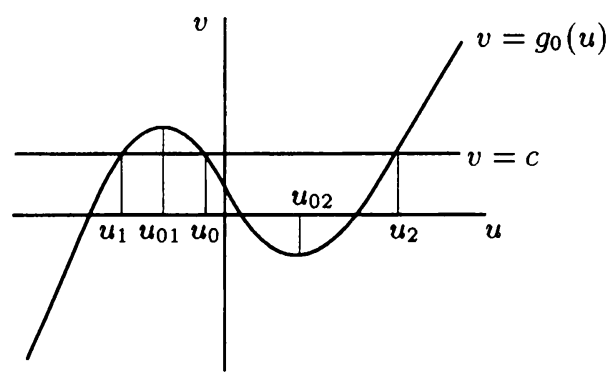

FIG. 2.1. The function $g_{0}$ 


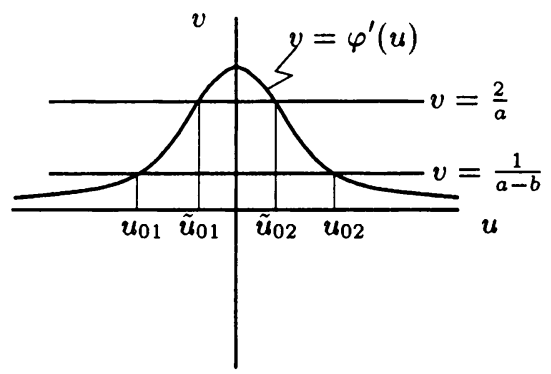

FIG. 2.2. The first derivative of $\varphi$

of $b<a-1$ given by

$$
c_{i}(b):=u_{0 i}(b)-(a-b) \varphi\left(u_{0 i}(b)\right), \quad i=1,2 .
$$

The next lemma provides a necessary condition for the existence of periodic orbits.

LEMma 2.2 (Nonexistence of periodic orbits). For $2 \geq a$, system (1.8) has no (nontrivial) periodic orbits.

Proof. For the divergence of the vector field given by system (1.8), it follows that

$$
\operatorname{div}(-u+a \varphi(u)-b v+c,-v+\varphi(u))=-2+a \varphi^{\prime}(u) .
$$

Using the properties of $\varphi^{\prime}$ (see Fig. 2) we get $-2+a \varphi^{\prime}(u)<0$ for $u \neq 0$ and $-2+a \varphi^{\prime}(u) \leq$ 0 for $u=0$. Bendixson's criterion (see [7, p. 44]) completes the proof.

Proposition 2.1. If $2<a$, then

(1) there are exactly two solutions $\tilde{u}_{01}, \tilde{u}_{02}, \tilde{u}_{01}<0<\tilde{u}_{02}=-\tilde{u}_{01}$, of

$$
2-a \varphi^{\prime}(u)=0
$$

and

(2) $u_{01}<\tilde{u}_{01}<0<\tilde{u}_{02}<u_{02}$, provided $b<\frac{a}{2}$, $u_{01}=\tilde{u}_{01}<0<\tilde{u}_{02}=u_{02}$, provided $b=\frac{a}{2}$,

where $u_{01}$ and $u_{02}$ are the solutions of Eq. (2.3).

Proof. Part 1 follows from the monotonicity of $\varphi^{\prime}$ on $(-\infty, 0]$ and $[0,+\infty)$ (see Fig. 2.2). For the proof of Part 2 we use that $b \leq \frac{a}{2}$ if and only if $a-b \geq \frac{a}{2}$. Then the monotonicity of $\varphi^{\prime}$ on $(-\infty, 0]$ and $[0,+\infty)$ yields Part 2 (see Fig. 2.2).

Proposition 2.2. If $2<a$, then $c_{2}<\tilde{c}_{2}<\tilde{c}_{1}<c_{1}$, provided $b<\frac{a}{2}$ and $c_{2}=\tilde{c}_{2}<\tilde{c}_{1}=$ $c_{1}$, provided $b=\frac{a}{2}$, where $\tilde{c}_{1}$ and $\tilde{c}_{2}$ are given by

$$
\tilde{c}_{i}:=g_{0}\left(\tilde{u}_{0 i}\right), \quad i=1,2
$$

and $c_{1}, c_{2}$ are as in Lemma 2.1 .

Proof. For $b<\frac{a}{2}$, it follows that (see Proposition 2.1)

$$
u_{01}<\tilde{u}_{01}<0<\tilde{u}_{02}<u_{02} .
$$


Because $g_{0}$ is strictly decreasing on $\left[u_{01}, u_{02}\right]$ we obtain $c_{2}<\tilde{c}_{2}<\tilde{c}_{1}<c_{1}$. If $b=\frac{a}{2}$, then $1-(a-b) \varphi^{\prime}(u)=0$ if and only if $2-a \varphi^{\prime}(u)=0$ and thus $c_{i}=\tilde{c}_{i}, i=1,2$.

Proposítion 2.3. Suppose $2<a$ and $b \leq \frac{a}{2}$. Then for $c \in\left(\tilde{c}_{2}, \tilde{c}_{1}\right)$, the system (1.8) has exactly three equilibria $\left(u_{1}, v_{1}\right),\left(u_{0}, v_{0}\right)$, and $\left(u_{2}, v_{2}\right)$ and it follows that

$$
u_{1}<\tilde{u}_{(01}<u_{0}<\tilde{u}_{02}<u_{2} .
$$

Proof. From Proposition 2.2 we know that $c_{2}<\tilde{c}_{2}<\tilde{c}_{1}<c_{1}$. Then Lemma 2.1 provides the existence of the three equilibria $\left(u_{1}, v_{1}\right),\left(u_{0}, v_{0}\right)$, and $\left(u_{2}, v_{2}\right)$ of (1.8), provided $c \in\left(\tilde{c}_{2}, \tilde{c}_{1}\right)$. By Proposition 2.1 we get $u_{01}<\tilde{u}_{01}<\tilde{u}_{02}<u_{02}$. This yields $u_{1}<\tilde{u}_{01}$ and $\tilde{u}_{02}<u_{2}$; see also Figures 2.1 and 2.2. Finally, the monotonicity of $g_{0}$ on $\left[u_{01}, u_{02}\right]$ and Eq. (2.1) imply that $\tilde{u}_{01}<u_{0}<\tilde{u}_{02}$.

The symmetrical case. Substitution of

$$
c=\frac{1}{2}(b-a)
$$

into system (1.8) yields

$$
\begin{aligned}
& \frac{d u}{d t}=-u+a \varphi(u)-b v+\frac{b-a}{2}, \\
& \frac{d v}{d t}=-v+\varphi(u) .
\end{aligned}
$$

From Lemma 2.1 we obtain

LEMMA 2.3 (Existence of equilibria in symmetrical case).

(1) If $1 \geq a-b$, then $(u, v)=\left(0, \frac{1}{2}\right)$ is the only equilibrium of $(2.10)$.

(2) If $1<a-b$, then there exist exactly three equilibria $\left(u_{1}, v_{1}\right),\left(u_{0}, v_{0}\right)$, and $\left(u_{2}, v_{2}\right)$ of $(2.10)$ and it follows that

$$
u_{1}<u_{0}=0<u_{2}=-u_{1} .
$$

Proof. $(u, v) \in \mathbb{R}^{2}$ is an equilibrium of (2.10) if and only if

$$
g_{0 . S}(u):=u-(a-b)\left(\varphi(u)-\frac{1}{2}\right)=0, \quad v=\varphi(u) .
$$

This yields the assertions of the lemma (see also the proof of Lemma 2.1).

REMARK 2.2. We call this case symmetrical because, under the condition (2.9), system (1.8) exhibits symmetry with respect to the equilibrium $\left(0, \frac{1}{2}\right)$. To be more precise, if $(u(t), v(t))$ is a solution of system $(2.10)$, then so is $(-u(t), 1-v(t))$.

Proposition 2.4. Suppose $2<a, 1<a-b$, and $c=\frac{1}{2}(b-a)$. Then there exists $\tilde{b} \in\left(\frac{a}{2}, a-1\right)$ such that

(1) $u_{1}<\tilde{u}_{01}<u_{0}=0<\tilde{u}_{02}<u_{2}$, provided $0<b<\tilde{b}$,

(2) $u_{1}=\tilde{u}_{01}<u_{0}=0<\tilde{u}_{02}=u_{2}$, provided $b=\tilde{b}$, and

(3) $\tilde{u}_{01}<u_{1}<u_{0}=0<u_{2}<\tilde{u}_{02}$, provided $\tilde{b}<b<a-1$.

Proof. Differentiating Eq. (2.11) with respect to $b$, one obtains

$$
\frac{d u}{d b} g_{0 S}^{\prime}(u)=-\left(\varphi(u)-\frac{1}{2}\right) \text {. }
$$

This implies that $u_{1}$ is a strictly increasing function of $b<a-1$ and that $u_{2}$ is a strictly decreasing function of $b<a-1$, while $u_{1}=u_{2}=0$ for $b=a-1$. 
Furthermore, from Proposition 2.3 and Lemma 2.3, we know that $u_{1}<\tilde{u}_{01}<u_{0}=$ $0<\tilde{u}_{02}<u_{2}$, if $b \leq \frac{a}{2}$. Then, the continuity and the monotonicity of $u_{1}$ and $u_{2}$ provide the existence of $\tilde{b}$ with the properties above, and thus the proof of the proposition is complete.

3. Liénard systems. The first aim of this section is to show how system (1.8) can be reduced to a system of the form:

$$
\begin{aligned}
& \frac{d x}{d t}=y-F(x), \\
& \frac{d y}{d t}=-g(x) .
\end{aligned}
$$

System (3.1) is referred to as a Liénard system. There exists an extensive literature on periodic solutions of the Liénard system; see for instance [15], [18], and [21]. Most results concern the nonexistence, existence, or uniqueness of limit cycles of system (3.1). After the reduction of system (1.8) to a Liénard system, we will state three lemmas that will appear to be useful in the sequel.

Let $(\bar{u}, \bar{v}) \in \mathbb{R}^{2}$ be an equilibrium of (1.8). We will use new coordinates

$$
\begin{aligned}
& x:=u-\bar{u}, \\
& y:=u-\bar{u}-b(v-\bar{v}) .
\end{aligned}
$$

Then from system (1.8) and Eq. (2.1) we get

$$
\begin{aligned}
& \frac{d x}{d t}=y-(2 x-a(\varphi(x+\bar{u})-\varphi(\bar{u}))) \\
& \frac{d y}{d t}=-(x-(a-b)(\varphi(x+\bar{u})-\varphi(\bar{u}))) .
\end{aligned}
$$

Clearly system (3.3) is of the form (3.1) with

$$
\begin{aligned}
F(x) & :=2 x-a(\varphi(x+\bar{u})-\varphi(\bar{u})), \\
g(x) & :=x-(a-b)(\varphi(x+\bar{u})-\varphi(\bar{u})) .
\end{aligned}
$$

Next let us state some lemmas that guarantee nonexistence or uniqueness of limit cycles for the Liénard system (3.1).

Lemma 3.1 (Nonexistence of limit cycles). Consider the Liénard system (3.1). Suppose that $F(x), g(x)$ are continuously differentiable functions on the interval $(\alpha, \beta)$ where $\alpha<0<\beta$. Assume that

(i) there exist $x_{1}, x_{2}$ with $\alpha<x_{1}<0<x_{2}<\beta$ such that

$$
\begin{aligned}
& g\left(x_{1}\right)=g(0)=g\left(x_{2}\right)=0, \\
& g(x)>0, \quad \text { for } x \in\left(x_{1}, 0\right) \cup\left(x_{2}, \beta\right), \\
& g(x)<0, \quad \text { for } x \in\left(\alpha, x_{1}\right) \cup\left(0, x_{2}\right) ;
\end{aligned}
$$




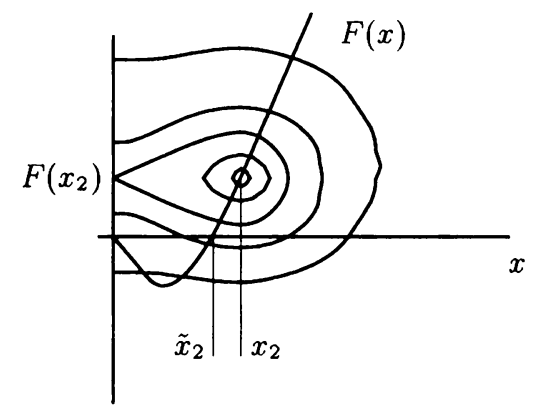

FIG. 3.1. Level sets of $\lambda(x, y) ; \kappa=-F\left(x_{2}\right), x \geq 0$

(ii) there exist $\tilde{x}_{1}, \tilde{x}_{2}$ with $x_{1} \leq \tilde{x}_{1}<0<\tilde{x}_{2} \leq x_{2}$ such that

$$
\begin{aligned}
& F\left(\tilde{x}_{1}\right)=F(0)=F\left(\tilde{x}_{2}\right)=0, \\
& F(x)>0, \quad \text { for } x \in\left(\tilde{x}_{1}, 0\right) \cup\left(\tilde{x}_{2}, \beta\right), \\
& F(x)<0, \quad \text { for } x \in\left(\alpha, \tilde{x}_{1}\right) \cup\left(0, \tilde{x}_{2}\right) .
\end{aligned}
$$

Then system (3.1) has no periodic orbits in the strip $\alpha<x<\beta$.

Proof. First we note that, under the above conditions, system (3.1) has three equilibria: $O(0,0), A\left(x_{2}, F\left(x_{2}\right)\right)$, and $A^{\prime}\left(x_{1}, F\left(x_{1}\right)\right)$. It is easy to check that $O$ is a saddle while $A$ and $A^{\prime}$ are antisaddles. Recall that an antisaddle is an equilibrium of which the product of the eigenvalues of the linearization matrix is positive. Therefore, limit cycles in system (3.1) either surround exactly one equilibrium ( $A$ or $A^{\prime}$ ) or exactly three equilibria. We will refer to the first type of limit cycles as "small" and to the latter type as "large".

The main tool we will need in the proof is the use of an energy function $\lambda(x, y)$, depending on a parameter $\kappa$ :

$$
\lambda(x, y)=\frac{1}{2}(y+\kappa)^{2}+G(x)
$$

where $G(x)=\int_{0}^{x} g(s) d s$.

Note that the level curves of $\lambda(x, y)$ are all closed. For the rate of change of trajectories of system (3.1) along such a curve we find

$$
\frac{d \lambda}{d t}=(y+\kappa) \frac{d y}{d t}+g(x) \frac{d x}{d t}=-g(x)(F(x)+\kappa) .
$$

First we will consider trajectories of system (3.1) in the half-plane $x \geq 0$. Now we choose $\kappa=-F\left(x_{2}\right)<0$. Then the level sets of $\lambda(x, y)$ for $x \geq 0$ are as depicted in Fig. 3.1. Note that all closed level sets of $\lambda(x, y)$ contain $A\left(x_{2}, F\left(x_{2}\right)\right)$.

It is easy to check that for $\kappa=-F\left(x_{2}\right)$, it follows from (3.6) that $\frac{d \lambda}{d t} \leq 0$, for $x \geq 0$. Therefore, any trajectory of system (3.1) intersects a level set of $\lambda(x, y), x \geq 0$, in the exterior-to-interior direction. This implies that system (3.1) has no small limit cycles surrounding $A$ because a small limit cycle surrounding $A$ has to be situated in $x>0$. Obviously $A$ is asymptotically stable. 


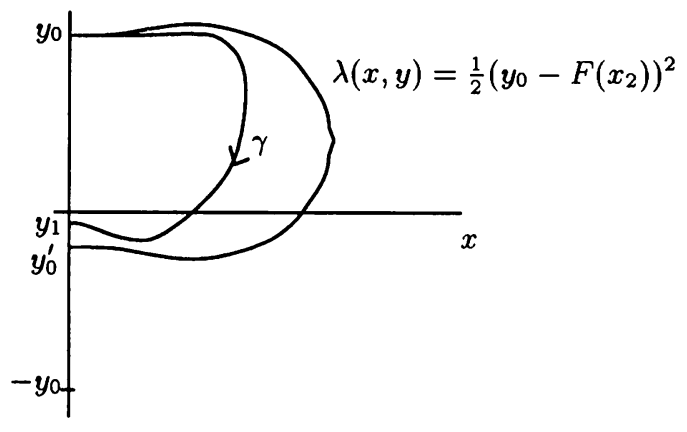

FIG. 3.2. The trajectory $\gamma$

Next we consider a trajectory $\gamma$ of system $(3.1), x \geq 0$, starting in $\left(0, y_{0}\right)$, where $y_{0}>-\kappa=F\left(x_{2}\right)>0$. Let $y_{0}^{\prime}$ be the $y$-coordinate of the intersection of the level set of $\lambda(x, y), \kappa=-F\left(x_{2}\right)$, with the $y$-axis below $y=-\kappa$, which also contains $\left(0, y_{0}\right)$. Note that this level set satisfies $\frac{1}{2}\left(y-F\left(x_{2}\right)\right)^{2}+G(x)=\frac{1}{2}\left(y_{0}-F\left(x_{2}\right)\right)^{2}$. It follows that

$$
y_{0}^{\prime}=-y_{0}+2 F\left(x_{2}\right)>-y_{0} \text {. }
$$

If $\gamma$ does not intersect the negative $y$-axis then $\gamma$ will have $A$ as its $\omega$-limit set. Therefore we will assume that $\gamma$ intersects the negative $y$-axis, say in $\left(0, y_{1}\right)$. Note that this implies that we assume $y_{0}^{\prime}<0$.

Because $\frac{d \lambda}{d t} \leq 0$ for $\kappa=-F\left(x_{2}\right), x \geq 0$, it follows that

$$
y_{1}>y_{0}^{\prime}>-y_{0}
$$

see Fig. 3.2.

To complete the proof we will consider trajectories of system (3.1) in the half-plane $x \leq 0$ and choose $\kappa=-F\left(x_{1}\right)>0$. Then in a similar way as in the case $x \geq 0$ one can prove that $A^{\prime}\left(x_{1}, F\left(x_{1}\right)\right)$ is asymptotically stable and not surrounded by a small limit cycle. We can also show that for a trajectory starting in $\left(0, y_{1}\right), y_{1}<-\kappa=F\left(x_{1}\right)<0$, crossing the positive $y$-axis, say in $\left(0, y_{2}\right)$, we have

$$
y_{2}<-y_{1} .
$$

Combining (3.8), (3.9) we find that $y_{2}<y_{0}$ and therefore there can be no limit cycles containing the three equilibria $O, A$, and $A^{\prime}$. This completes the proof.

LEmma 3.2 (Uniqueness of limit cycles). Consider the Liénard system (3.1) on $\mathbb{R}^{2}$, where the functions $g(x)$ and $F(x)$ are continuously differentiable functions. Assume that

(i) $g(-x)=-g(x)$ and $F(-x)=-F(x)$;

(ii) $g(x)>0$ for $x>0$;

(iii) there exists a constant $\zeta>0$ such that $F(0)=F(\zeta)=0$ and $F(x)<0$ for $x \in(0, \zeta)$;

(iv) for $x>\zeta, F(x)$ is monotonically increasing and $\lim _{x \rightarrow \infty} F(x)=\infty$.

Then system (3.1) has exactly one limit cycle. The limit cycle is stable and hyperbolic. 
Lemma 3.2 is basically due to Liénard [12]; sce also [11]. An alternative proof of this lemma, which also concludes on the hyperbolicity of the limit cycle, can be found in [13].

Lemma 3.3 (Uniqueness of limit cycles). Consider the Liénard system (3.1) where the functions $F(x), g(x)$ are continuously differentiable on the open interval $(\alpha, \beta)$. Define $f(x) \equiv \frac{d}{d x} F(x)$.

Suppose:

(i) there exists a $\xi \in(\alpha, \beta)$ such that $(x-\xi) g(x)>0$ for $x \in(\alpha, \beta) \backslash\{\xi\}$;

(ii) there exists a $\mu \in(\alpha, \beta)$ such that $(x-\mu) f(x)>0$ for $x \in(\alpha, \beta) \backslash\{\mu\}$.

Then if

(a) there exists $\kappa \in \mathbb{R}$ such that $f(x)-\kappa g(x)$ does not change sign, then system (3.1) has no limit cycles in the strip $\alpha<x<\beta$;

(b) if $\mu<\xi$ and $\frac{d}{d x}\left(\frac{f(x)}{g(x)}\right)<0$ for $x \in(\alpha, \mu) \cup(\xi, \beta)$, then system (3.1) has at most one limit cycle in the strip $\alpha<x<\beta$, which is unstable and hyperbolic if it exists.

Implication (a) follows after an application of Dulac's criterion with Dulac function $B(x, y)=\exp (-\kappa y)$ to (3.1). Implication (b) follows from a modification of a theorem by Zhang Zhi-fen [19], [20].

4. Application to the neuron model. Now we are in a position to state and to prove the results mentioned in the introduction on the number of periodic orbits of system (1.8). The first theorem provides conditions that guarantee the nonexistence of periodic orbits in the case that system (1.8) possesses three equilibria.

THEOREM 4.1 (Nonexistence of periodic orbits).

(1) For $2 \geq a$, the system (1.8) has no periodic orbits.

(2) Suppose $2<a$ and $b \leq \frac{a}{2}$. Then for $c \in\left(\tilde{c}_{2}, \tilde{c}_{1}\right)$, the system (1.8) has no periodic orbits, where $\tilde{c}_{1}$ and $\tilde{c}_{2}$ are given by Eq. (2.8).

Proof. The first part follows from Lemma 2.2. The proof of the second part is an application of Lemma 3.1. In Sec. 3 we have demonstrated that system (1.8) can be transformed to a Liénard system with

$$
\begin{aligned}
F(x) & :=2 x-a(\varphi(x+\bar{u})-\varphi(\bar{u})), \\
g(x) & :=x-(a-b)(\varphi(x+\bar{u})-\varphi(\bar{u})) .
\end{aligned}
$$

From Proposition 2.3 we know that for $c \in\left(\tilde{c}_{2}, \tilde{c}_{1}\right)$, system (1.8) has exactly three equilibria $\left(u_{1}, v_{1}\right),\left(u_{0}, v_{0}\right)$, and $\left(u_{2}, v_{2}\right)$ with $u_{1}<u_{0}<u_{2}$. We set $\bar{u}=u_{0}$ and we study the properties of the functions $g$ and $F$.

We start with the function $g(x)$. First note that $2<a$ and $b \leq \frac{a}{2}$ imply $1<a-b$. Now, from $1<a-b$ it follows (see proof of Lemma 2.1): $g$ is strictly increasing on $\left(-\infty, u_{01}-u_{0}\right]$ and $\left[u_{02}-u_{0},+\infty\right)$; and $g$ is strictly decreasing on $\left[u_{01}-u_{0}, u_{02}-u_{0}\right]$, where $u_{01}$ and $u_{02}$ are given by Eq. (2.3). Since $u_{01}-u_{0}<0<u_{02}-u_{0}, g(0)=0$, and $\lim _{x \rightarrow \pm \infty} g(x)= \pm \infty$, this implies the existence of $x_{1}$ and $x_{2}, x_{1}<0<x_{2}$ (note, 


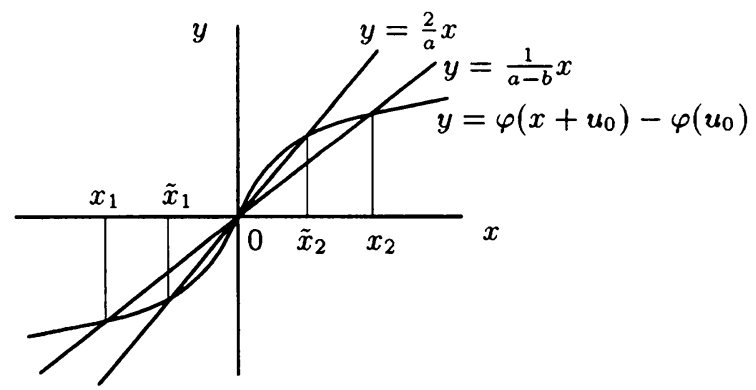

FIG. 4.1. The zeros of the functions $F$ and $g$

$\left.x_{i}=u_{i}-u_{0}, i=1,2\right)$ such that

$$
\begin{gathered}
g\left(x_{1}\right)=g(0)=g\left(x_{2}\right)=0, \\
g(x)>0, \quad \text { for } x \in\left(x_{1}, 0\right) \cup\left(x_{2}, \infty\right), \\
g(x)<0, \quad \text { for } x \in\left(-\infty, x_{1}\right) \cup\left(0, x_{2}\right) .
\end{gathered}
$$

Note that we apply Lemma 3.1 with $a=-\infty$ and $\beta=\infty$.

Now let us consider the function $F$. The first derivative of $F$ satisfies

$$
F^{\prime}(x)=2-a \varphi^{\prime}\left(x+u_{0}\right) .
$$

Since $2<a$ and $c \in\left(\tilde{c}_{2}, \tilde{c}_{1}\right)$ (see Propositions 2.1 and 2.3) this yields: $F$ is strictly increasing on $\left[-\infty, \tilde{u}_{01}-u_{0}\right]$ and $\left[\tilde{u}_{02}-u_{0},+\infty\right)$; and $F$ is strictly decreasing on $\left[\tilde{u}_{01}-\right.$ $\left.u_{0}, \tilde{u}_{02}-u_{0}\right]$, where $\tilde{u}_{01}$ and $\tilde{u}_{02}$ are given by Eq. (2.7). Then, since $\tilde{u}_{01}-u_{0}<0<$ $\tilde{u}_{02}-u_{0}, F(0)=0$, and $\lim _{x \rightarrow \pm \infty} F(x)= \pm \infty$, this provides the existence of $\tilde{x}_{1}$ and $\tilde{x}_{2}$, $\tilde{x}_{1}<0<\tilde{x}_{2}$ such that

$$
\begin{gathered}
F\left(\tilde{x}_{1}\right)=F(0)=F\left(\tilde{x}_{2}\right)=0, \\
F(x)>0, \quad \text { for } x \in\left(\tilde{x}_{1}, 0\right) \cup\left(\tilde{x}_{2}, \infty\right), \\
F(x)<0, \quad \text { for } x \in\left(-\infty, \tilde{x}_{1}\right) \cup\left(0, \tilde{x}_{2}\right) .
\end{gathered}
$$

In order to be able to apply Lemma 3.1, we still need to prove that $x_{1} \leq \tilde{x}_{1}<0<\tilde{x}_{2} \leq x_{2}$. Because $F(x)=0$ if and only if $\frac{2}{a} x-\left(\varphi\left(x+u_{0}\right)-\varphi\left(u_{0}\right)\right)=0$, and $g(x)=0$ if and only if $\frac{1}{a-b} x-\left(\varphi\left(x+u_{0}\right)-\varphi\left(u_{0}\right)\right)=0$, from $b \leq \frac{a}{2}$ we get $x_{1} \leq \tilde{x}_{1}<0<\tilde{x}_{2} \leq x_{2}$ (see Fig. 4.1). This completes the proof of the theorem.

Theorem 4.2 (Uniqueness of periodic orbits). Suppose $2<a, 1>a-b$, and $c=\frac{1}{2}(b-$ $a$ ). Then system (1.8) has exactly one limit cycle, which is stable and hyperbolic.

Proof. The theorem is a consequence of Lemma 3.2. Again we will work with the Liénard system (3.1) where (4.1) holds and in addition $c=\frac{1}{2}(b-a)$.

From Lemma 2.3 we know that when $1>a-b$ and $c=\frac{1}{2}(b-a)$, the unique equilibrium of system $(1.8)$ is $(u, v)=\left(0, \frac{1}{2}\right)$. We set $\bar{u}=0$ and we study the properties of the functions $g$ and $F$. It is easy to see that both $g$ and $F$ are symmetric, that is, $g(-x)=$ $-g(x)$ and $F(-x)=-F(x)$. From $1>a-b$ it follows (see proof of Lemma 2.1) that $g$ is strictly increasing on $\mathbb{R}$. Then, because $g(0)=0$, we get $x g(x)>0$, for $x \neq 0$. 
Now let us consider the function $F$. As in the proof of Theorem 4.1, the assumption $2<a$ yields the existence of $\tilde{x}_{1}$ and $\tilde{x}_{2}, \tilde{x}_{1}<0<\tilde{x}_{2}$, such that

$$
\begin{gathered}
F\left(\tilde{x}_{1}\right)=F(0)=F\left(\tilde{x}_{2}\right)=0, \\
F(x)>0, \quad \text { for } x \in\left(\tilde{x}_{1}, 0\right) \cup\left(\tilde{x}_{2}, \infty\right), \\
F(x)<0, \quad \text { for } x \in\left(-\infty, \tilde{x}_{1}\right) \cup\left(0, \tilde{x}_{2}\right) .
\end{gathered}
$$

Note that $\lim _{x \rightarrow \pm \infty} F(x)= \pm \infty$, and because of the symmetry of $F, \tilde{x}_{2}=-\tilde{x}_{1}$. Finally, it is easy to check that $F$ is strictly increasing on $\left(-\infty, \tilde{x}_{1}\right)$ and $\left(\tilde{x}_{2}, \infty\right)$. The theorem follows from Lemma 3.2.

Theorem 4.3. Suppose $2<a, 1<a-b$, and $c=\frac{1}{2}(b-a)$. Then, if $b \in[0, a / 2] \cup[\tilde{b}, a-1)$, system (1.8) has no small limit cycles, and, if $b \in(a / 2, \tilde{b})$, system (1.8) has at most one small cycle around each of the equilibria $\left(u_{1}, v_{1}\right)$ and $\left(u_{2}, v_{2}\right)$, with $\tilde{b}$ as in Proposition 2.4 .

Proof. We will use Lemma 3.3 after transforming system (1.8) to a Liénard system. If we take

$$
\bar{u}=0, \quad \bar{v}=\frac{1}{2},
$$

then for $c=\frac{1}{2}(b-a)$, the transformation (3.2) reduces system (1.8) to

$$
\begin{aligned}
& \frac{d x}{d t}=y-F(x), \\
& \frac{d y}{d t}=-g(x),
\end{aligned}
$$

with

$$
\begin{gathered}
F(x)=2(x+2 d \varphi(x)-d), \\
g(x)=x+2 \eta \varphi(x)-\eta,
\end{gathered}
$$

where

$$
d=-\frac{1}{4} a, \quad \eta=-\frac{1}{2}(a-b), \quad \varphi(x):=\frac{1}{1+\exp (-4 x)} .
$$

It follows from $2<a, 1<a-b$ that

$$
d<-\frac{1}{2}, \quad \eta<-\frac{1}{2} .
$$

Note that $F(-x)=-F(x)$ and $g(-x)=-g(x)$ and therefore system (4.4) is symmetric with respect to the origin.

As usual we define $f(x) \equiv \frac{d}{d x}(F(x))=2\left(1+2 d \varphi^{\prime}(x)\right)$.

For the proof of Theorem 4.3 we will need the following lemma.

LEMMA 4.1. For all $\kappa>0$, the function $f(x)-\kappa g(x)=0$ has at most two positive zeros, multiplicity taken into account.

The proof of Lemma 4.1 will be stated later.

If $0<b<\frac{a}{2}$, Theorem 4.1 yields the nonexistence of any periodic orbit. For $\frac{a}{2}<b<$ $a-1$, we will apply Lemma 3.3 to the system (4.4) in the strip $(0, \infty)$, i.e., $\alpha=0, \beta=\infty$. 


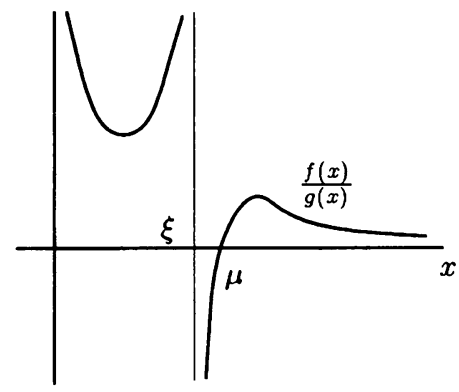

FIG. 4.2. The function $\frac{f(x)}{g(x)}, \mu>\xi$

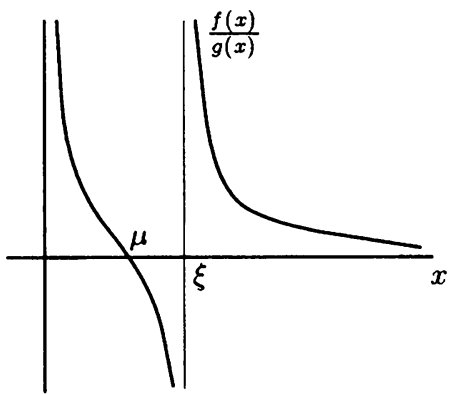

FIG. 4.3. The function $\frac{f(x)}{g(x)}, \mu<\xi$

Clearly condition (i) is satisfied, where $\xi$ is the unique positive zero of $g(x)$. Note that $\xi=u_{2}$. Furthermore, for $d<-\frac{1}{2}, f(x)$ has a unique positive zero $\mu$. Note that $\mu=\tilde{u}_{02}$. First we consider the case $\tilde{b}<b<a-1$. This provides $\mu>\xi$, i.e., the equilibrium $(\xi, F(\xi))$ is unstable. Let us study the graph of $\frac{f(x)}{g(x)}$ for this case. Because this function has vertical asymptotes at $x=0, x=\xi$ and a horizontal asymptote at $y=0$, it follows from application of Lemma 4.1 that the graph of $\frac{f(x)}{g(x)}$ is as depicted in Fig. 4.2.

Obviously, there exists a constant $\kappa>0$ such that $\frac{f(x)}{g(x)}=\kappa$, and hence $f(x)-\kappa g(x)=0$ has no positive zeros. It follows from Lemma 3.3, implication (a), that system (4.4) has no limit cycles in the strip $0<x<\infty$. Because system (4.4) is symmetric with respect to the origin, this implies that system (4.4) has no small limit cycles in the whole phase plane.

Next we consider the case $\frac{a}{2}<b<\tilde{b}$. It follows that $\mu<\xi$, i.e., $(\xi, F(\xi))$ is asymptotically stable. Again we study the graph of $\frac{f(x)}{g(x)}$, and use the fact that $x=0, x=\xi$, and $y=0$ are asymptotes; see Fig. 4.3.

It follows from Lemma 4.1 that, on the intervals $(0, \mu)$ and $(\xi, \infty), \frac{d}{d x}\left(\frac{f(x)}{g(x)}\right)<0$ holds. It follows from Lemma 3.3, implication (b), that system (4.4) has at most one small limit cycle in the strip $0<x<\infty$. If it exists, it is unstable and hyperbolic. By symmetry, system (4.4) has at most two small limit cycles in the whole phase plane. 
Finally, we consider the case $b=\tilde{b}$. This yields $\mu=\xi$, i.e., the situation where the Hopf bifurcation occurs. We will use Lemma 3.3, implication (a), to prove the nonexistence of a small limit cycle in this case. Let $h(x)=f(x)-\kappa_{0} g(x)$ with $\kappa_{0}=\frac{f^{\prime}(\xi)}{g^{\prime}(\xi)}$. Then $h(x)$ has a multiple zero at $x=\xi$. According to Lemma 4.1, the zero $x=\xi$ has multiplicity two while there are no other positive zeros. Hence by Lemma 3.3, implication (a), system (4.4) has no small limit cycles in the strip $0<x<\infty$. Again by symmetry, there are no small limit cycles in the whole phase plane for this case. This finishes the proof of Theorem 4.3.

Proof of Lemma 4.1. Define

$$
h(x)=f(x)-\kappa g(x)=2+\frac{16 d \exp (-4 x)}{(1+\exp (-4 x))^{2}}-\kappa\left(x+\frac{2 \eta}{1+\exp (-4 x)}-\eta\right) .
$$

Substitution of

$$
\exp (-4 x)=y
$$

yields

$$
h^{*}(y)=h(x(y))=2+\frac{16 d y}{(1+y)^{2}}-\kappa\left(-\frac{1}{4} \ln (y)+\frac{2 \eta}{1+y}-\eta\right) .
$$

Note that because $x \in(0, \infty)$, it follows from (4.8) that $y \in(0,1)$.

From (4.9) it follows that

$$
\begin{aligned}
\frac{d h^{*}(y)}{d y} & =\frac{\kappa y^{3}+(8 \kappa \eta+3 \kappa-64 d) y^{2}+(64 d+3 \kappa+8 \kappa \eta) y+\kappa}{4 y(1+y)^{3}} \\
& \equiv \frac{h_{1}(y)}{4 y(1+y)^{3}} .
\end{aligned}
$$

Because $h_{1}(0)>0, h_{1}( \pm \infty)= \pm \infty$, and $h_{1}(1)=8 \kappa(1+2 \eta)<0$, it follows that $h_{1}(y)$ has a unique zero $y_{1}$ on $(0,1)$.

From (4.10) it follows that

$$
\begin{aligned}
\frac{d^{2} h^{*}(y)}{d y^{2}} & =-\frac{\kappa y^{4}+(4 \kappa-128 d+16 \kappa \eta) y^{3}+(256 d+6 \kappa+16 \kappa \eta) y^{2}+4 \kappa \eta y+\kappa}{4 y^{2}(1+y)^{4}} \\
& \equiv-\frac{h_{2}(y)}{4 y^{2}(1+y)^{4}} .
\end{aligned}
$$

Because $h_{2}(0)>0, h_{2}^{\prime}(0)>0, h_{2}( \pm \infty)=\infty$, and $h_{2}(1)=128 d+16 \kappa(1+2 \eta)<0$, it follows that $h_{2}(y)$ has a unique zero $y_{2}$ on $(0,1)$.

Finally, we need information about $h^{*}(y)$ on the end-points $y=0, y=1$. One can prove that

$$
\lim _{y \downarrow 0} h^{*}(y)=-\infty, \quad h^{*}(1)=1+2 d<0 .
$$

Note that for $0<y<1$, we have $\operatorname{sgn}\left(\frac{d h^{*}}{d y}\right)=\operatorname{sgn}\left(h_{1}(y)\right)$ and $\operatorname{sgn}\left(\frac{d^{2} h^{*}}{d y^{2}}\right)=\operatorname{sgn}\left(-h_{2}(y)\right)$.

First we prove that $y_{1}<y_{2}$. Suppose that $y_{1} \geq y_{2}$. Then for $y \in\left(0, y_{2}\right), h^{*}(y)$ is increasing with negative second derivative. At the inflection point $y=y_{2}$ the second derivative becomes positive; so $\frac{d h^{*}}{d y}$ cannot become zero at $y=y_{1}$, which is a contradiction.

Let us draw in one figure $h_{1}$ and $-h_{2}$; see Fig. 4.4. 


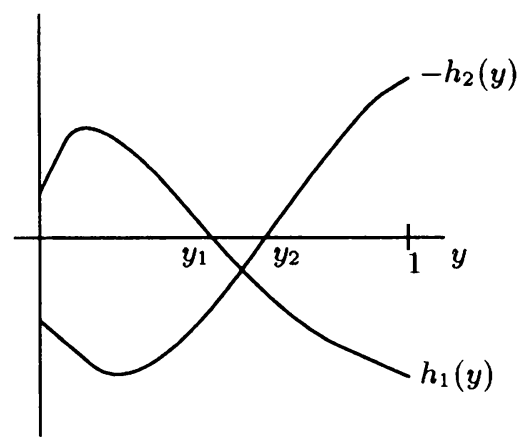

FIG. 4.4. The functions $h_{1}(y)$ and $-h_{2}(y)$

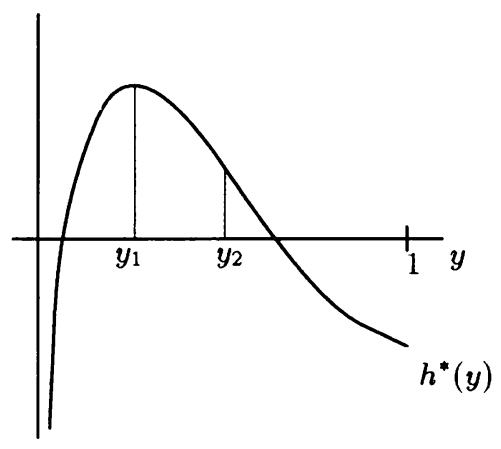

FIG. 4.5. The function $h^{*}(y)$

From Fig. 4.4 and (4.12) it can be deduced that $h^{*}(y)$ has at most two positive zeros for $0<y<1$; see also Fig. 4.5.

Because $h(x)$ and $h^{*}(y)$ are related through the bijection (4.8) it follows that $h(x)$ has at most two positive zeros. This finishes the proof of Lemma 4.1.

REMARK 4.1. The results stated in Theorems 4.1 and 4.2 easily can be generalized for a wide class of transfer functions $\varphi(u)$, with the same properties as the function we have chosen in (1.2). In fact, for any $\varphi \in C^{1}$ satisfying $\lim _{u \rightarrow-\infty} \varphi(u)=0, \lim _{u \rightarrow \infty} \varphi(u)=$ $C<\infty, \varphi^{\prime}(u)>0$, and $\varphi^{\prime}(-u)=\varphi^{\prime}(u)$, one can prove that results similar to Theorems 4.1 and 4.2 are valid. The generalization of Theorem 4.3 is more difficult because we have to verify the conditions of Lemma 3.3 for every individual choice of $\varphi$. As an example we mention that it can be shown that for $\varphi(u)=\frac{1+u+|u|}{1+|u|}$, the analogue of Theorem 4.3 holds true.

5. Conclusions. In this work, we have studied the number of periodic solutions of a planar dynamical system modelling the neural activity of a network consisting of two neurons. Our results provide relationships between the parameters of the system, which can be, in some cases, biologically interpreted (cf. [6]). For instance, if the positive feedback $a=q_{11}$ is so much stronger than the negative feedback $b=q_{12} q_{21}$ that $a>b+1$, 
then for stimulus configurations with $c=e_{1}-q_{12} e_{2} \in\left(\tilde{c}_{2}, \tilde{c}_{2}\right)$ (see Theorem 4.1) the network considered here is convergent, i.e., every trajectory converges to some equilibrium as $t$ goes to infinity (see [8]). In this case the neural network considered cannot have periodic activity. Convergent neural networks can be viewed as models for associative memory (see $[1,8,10,9]$ ). The stable equilibria are the stored memories. System (1.8) with $(b, c) \in A$ (see Fig. 1.3) provides a model for a simple associative memory with two stored memories, i.e., the two stable equilibria (see Fig. 1.6.A).

On the other hand, if the negative feedback $b=q_{12} q_{21}$ dominates such that $b>\frac{a}{2}$, provided that the positive feedback is strong enough such that $a=q_{11}>2$, the network is oscillatory, i.e., every trajectory tends asymptotically to a periodic (perhaps stationary) orbit (see [8]). Notice that (1.8) with $a \leq 2$ cannot have periodic activity (see Theorem 4.1). Rhythmic activity has been observed in several neurophysiological experiments and seems to play an important role in the information processing in the brain (see $[1,5])$. Our model shows that both strong interaction between excitatory and inhibitory neurons and strong recurrent excitation can give rise to oscillatory dynamics. System (1.8) with $(b, c) \in N$ (see Fig. 1.3 and Fig. 1.6.N) possesses a unique stable periodic orbit. Note that periodic oscillations are the only sustained oscillations that can be simulated by planar systems of ordinary differential equations.

Acknowledgments. We thank the referee for the helpful suggestions and comments.

\section{REFERENCES}

[1] B. Baird, Nonlinear dynamics of pattern formation and pattern recognition in the rabbit olfactory bulb, Physica 22D, 150-175 (1986)

[2] R. M. Borisyuk and A. B. Kirillov, Bifurcation analysis of a neural network model, Biol. Cybern. 66, 319-325 (1992)

[3] J. D. Cowan and G. B. Ermentrout, Some Aspects of the "Eigenbehavior" of Neural Nets, in S. A. Levin: Studies in Mathematical Biology Part I: Cellular Behavior and the Development of Pattern, Studies in Mathematics, vol. 15, Mathematical Association of America, Washington, DC, 1978, pp. $67-117$

[4] F. Dumortier, R. Roussarie, and J. Sotomayor, Generic 3-Parameter Families of Planar Fields, Unfoldings of Saddle, Focus and Elliptic Singularities with Nilpotent Linear Parts, in F. Dumortier, R. Roussarie, J. Sotomayor and H. Zoladek: Bifurcations of Planar Vector Fields: Nilpotent Singularities and Abelian Integrals, Lecture Notes in Mathematics 1480, Springer-Verlag, Berlin, 1991

[5] W. J. Freeman, Tutorial on neurobiology: From single neurons to brain chaos, International Journal of Bifurcations and Chaos 2, 441-482 (1992)

[6] F. Giannakopoulos and O. Oster, Bifurcation properties of a planar system modelling neural activity, Planar Nonlinear Dynamical Systems (Delft, 1995), Differential Equations and Dynamical Systems 5, 229-242 (1997)

[7] J. Guckenheimer and P. Holmes, Nonlinear Oscillations, Dynamical Systems and Bifurcation of Vector Fields, Springer-Verlag, New York, 1983

[8] M. W. Hirsch, Convergent Activation Dynamics in Continuous Time Networks, Neural Networks 2, 331-349 (1989)

[9] F. C. Hoppensteadt and E. M. Izhikevich, Weakly Connected Neural Networks, Springer-Verlag, New York, 1997

[10] J. J. Hopfield, Neural Networks and Physical Systems with Emergent Collective Computational Abilities, Proceedings of the National Academy of Sciences, USA 79, 2554-2558 (1982)

[11] N. Levinson and O. K. Smith, A general equation for relaxation oscillation, Duke Math. Journal $\mathbf{9}$, $382-403(1942)$ 
[12] A. Liénard, Étude des oscillations entretenues, Rev. Gen. d'Electricité, XXIII, 1928, pp. 901-946 [in French]

[13] R. E. Kooij and Sun Jianhua, A note on "Uniqueness of limit cycles in a Liénard type system", J. Math. Anal. Appl. 208, 260-276 (1997)

[14] J. G. Nicholls, A. R. Martin, and B. G. Wallace, From neuron to brain, Sinauer Associates Inc. Publishers, Sunderland, Massachusetts, USA, 1992

[15] G. Sansone and R. Conti, Nonlinear Differential Equations, Macmillan, New York, 1964

[16] G. M. Shepherd, Neurobiology, Oxford University Press, New York, 1994

[17] H. R. Wilson and J. D. Cowan, Excitatory and inhibitory interactions in localized populations of model neurons, Biophysical J. 12, 1-24 (1972)

[18] Ye Yan-Qian, Theory of limit cycles, Transl. of Math. Monographs, Vol. 66, Amer. Math. Soc., Providence, Rhode Island, 1986

[19] Zhang Zhi-fen, On the uniqueness of limit cycles of certain equations of nonlinear oscillations, Dokl. Akad. Nauk SSSR 119, 659-662 (1958) [in Russian]

[20] Zhang Zhi-fen, Proof of the uniqueness theorem of limit cycles of generalized Liénard equations, Appl. Anal. 23, 63-76 (1986)

[21] Zhang Zhi-fen, Ding Tong-ren, Huang Wen-zao, and Dong Zhen-xi, Qualitative Theory of Differential Equations, Translations of Math. Monographs, Vol. 101, Amer. Math. Soc., Providence, RI, 1992 